Commun. Korean Math. Soc. 19 (2004), No. 3, pp. 569-576

\title{
GENERALIZATION OF WATSON'S THEOREM FOR DOUBLE SERIES
}

\author{
Yong Sup Kim, Aruun K. Rathie, \\ Chan Bong Park and Chang Hyun Lee
}

\begin{abstract}
In 1965, Bhatt and Pandey obtained the Watson's theorem for double series by using Dixon's theorem on the sum of a ${ }_{3} F_{2}$. The aim of this paper is to derive twenty three results for double series closely related to the Watson's theorem for double series obtained by Bhatt and Pandey. The results are derived with the help of twenty three summation formulas closely related to the Dixon's theorem on the sum of a ${ }_{3} F_{2}$ obtained in earlier work by Lavoie, Grondin, Rathie and Arora.
\end{abstract}

\section{Introduction}

The generalized Kampé de Fériet function introduced by Srivastava and Panda [5] is defined and represented in the following manner:

$$
\begin{aligned}
& F_{l: m ; n}^{p: q ; k}\left[\begin{array}{c}
\left(a_{p}\right):\left(b_{q}\right) ;\left(c_{k}\right) \\
\left(\alpha_{l}\right):\left(\beta_{m}\right) ;\left(\gamma_{n}\right)
\end{array} \mid x, y\right] \\
= & \sum_{r, s=0}^{\infty} \frac{\prod_{j=1}^{p}\left(a_{j}\right)_{r+s} \prod_{j=1}^{q}\left(b_{j}\right)_{r} \prod_{j=1}^{k}\left(c_{j}\right)_{s}}{\prod_{j=1}^{l}\left(\alpha_{j}\right)_{r+s} \prod_{j=1}^{m}\left(\beta_{j}\right)_{r} \prod_{j=1}^{n}\left(\gamma_{j}\right)_{s}} \frac{x^{r}}{r !} \frac{y^{s}}{s !}
\end{aligned}
$$

where, for convergence,

(i) $p+q<l+m+1, p+k<l+n+1,|x|<\infty,|y|<\infty$, or

Received October 16, 2003.

2000 Mathematics Subject Classification: Primary 33C70, Secondary 33C05, $33 \mathrm{C} 20$.

Key words and phrases: the generalized Kampé de Fériet function, double series, Watson's theorem, Dixon's theorem.

The third author was supported by the Research Fund of Wonkwang University in 2004. 
570 Yong Sup Kim, Arjun K. Rathie, Chan Bong Park and Chang Hyun Lee

(ii) $p+q=l+m+1, p+q=l+n+1$, and

$$
\begin{cases}|x|^{\frac{1}{p-l}}+|y|^{\frac{1}{p-l}}<1, & \text { if } p>l \\ \max \{|x|,|y|\}<1, & \text { if } p \leq l\end{cases}
$$

(1.1) reduces to the Kampé de Fériet function [3] in the special case $q=k$ and $m=n$.

In 1965, Bhatt and Pandey [2] obtained the Watson's theorem for double series by using Dixon's theorem on the sum of a ${ }_{3} F_{2}$ with unit argument.

In 1994, Lavoie, Grondin, Rathie and Arora [ 4 ] generalized the Dixon's theorem which is given in the next section and they have obtained twenty three results closely related to the Dixon's theorem. In the same paper, they have obtained a large number of limiting cases of their results.

The aim of this paper is to derive twenty three results for the double series, in the form of a single result, closely related to the Watson's theorem for the following double series obtained earlier by Bhatt and Pandey [2]:

$$
\begin{aligned}
& F_{2: 0 ; 0}^{2: 1 ; 1}\left[\begin{array}{r}
a_{1}, a_{2}: b_{1} ; b_{1}^{\prime} \\
\frac{1}{2}\left(1+a_{1}+a_{2}\right), 2 b_{1}+2 b_{1}^{\prime}:-;-1,1
\end{array}\right] \\
& =\frac{\Gamma\left(\frac{1}{2}\right) \Gamma\left(b_{1}+b_{1}^{\prime}+\frac{1}{2}\right) \Gamma\left(\frac{1}{2}+\frac{1}{2} a_{1}+\frac{1}{2} a_{2}\right) \Gamma\left(\frac{1}{2}-\frac{1}{2} a_{1}-\frac{1}{2} a_{2}+b_{1}+b_{1}^{\prime}\right)}{\Gamma\left(\frac{1}{2} a_{1}+\frac{1}{2}\right) \Gamma\left(\frac{1}{2} a_{2}+\frac{1}{2}\right) \Gamma\left(\frac{1}{2}+b_{1}+b_{1}^{\prime}-\frac{1}{2} a_{1}\right) \Gamma\left(\frac{1}{2}+b_{1}+b_{1}^{\prime}-\frac{1}{2} a_{2}\right)} .
\end{aligned}
$$

\section{Results required}

The following results will be required in our present investigation.

Transformation formula [1]:

$$
\begin{aligned}
& { }_{3} F_{2}\left[\begin{array}{r}
a, b, c \\
e, f
\end{array}\right] \\
= & \frac{\Gamma(e) \Gamma(f) \Gamma(s)}{\Gamma(a) \Gamma(s+b) \Gamma(s+c)}{ }_{3} F_{2}\left[\begin{array}{r}
e-a, f-a, s \\
s+b, s+c
\end{array} \mid 1\right]
\end{aligned}
$$

where $s=e+f-a-b-c$ and $\Re e(s)>0$. 
Generalized Dixon's theorem [4]:

$(2.2)$

$$
\begin{aligned}
& { }_{3} F_{2}\left[\begin{array}{r}
a, b, c \mid 1 \\
1+a-b+i, 1+a-c+i+j
\end{array}\right] \\
= & \frac{2^{-2 c+i+j} \Gamma(1+a-b+i) \Gamma(1+a-c+i+j) \Gamma\left(b-\frac{i}{2}-\frac{|i|}{2}\right)}{\Gamma(a-2 c+i+j+1) \Gamma(a-b-c+i+j+1)} \\
& \times \frac{\Gamma\left(c-\frac{1}{2}(i+j+|i+j|)\right)}{\Gamma(b) \Gamma(c)} \\
& \times\left\{A_{i, j} \frac{\Gamma\left(\frac{1}{2} a-c+\frac{1}{2}+\left[\frac{i+j+1}{2}\right]\right) \Gamma\left(\frac{1}{2} a-b-c+1+i+\left[\frac{j+1}{2}\right]\right)}{\Gamma\left(\frac{1}{2} a+\frac{1}{2}\right) \Gamma\left(\frac{1}{2} a-b+1+\left[\frac{i}{2}\right]\right)}\right. \\
& \left.+B_{i, j} \frac{\Gamma\left(\frac{1}{2} a-c+1+\left[\frac{i+j}{2}\right]\right) \Gamma\left(\frac{1}{2} a-b-c+\frac{3}{2}+i+\left[\frac{j}{2}\right]\right)}{\Gamma\left(\frac{1}{2} a\right) \Gamma\left(\frac{1}{2} a-b+\frac{1}{2}+\left[\frac{i+1}{2}\right]\right)}\right\}
\end{aligned}
$$

for $i=0, \pm 1, \pm 2, \pm 3$ and $j=0,1,2,3$. Also $\Re e(a-2 b-2 c)>-2-2 i-j$. $[x]$ is the greatest integer less than or equal to $x$ and its absolute value is denoted by $|x|$. The coefficients $A_{i, j}$ and $B_{i, j}$ appear in the tables at the end of the paper.

\section{Main result}

The result to be proved is

$$
\begin{aligned}
& F_{2: 0 ; 0}^{2: 1 ; 1}\left[2 b_{1}+2 b_{1}^{\prime}+j, \frac{1}{2}\left(1+a_{1}+a_{2}+i\right):-;-a_{2} ; b_{1}^{\prime} \mid 1,1\right] \\
= & \frac{\Gamma\left(2 b_{1}+2 b_{1}^{\prime}+j\right) \Gamma\left(\frac{a_{1}}{2}+\frac{a_{2}}{2}+\frac{i}{2}+\frac{1}{2}\right) 2^{-2 b_{1}-2 b_{1}^{\prime}+a_{1}+a_{2}-j-1}}{\Gamma\left(a_{1}\right) \Gamma\left(a_{2}\right) \Gamma\left(b_{1}+b_{1}^{\prime}+j\right) \Gamma\left(\frac{1}{2}-\frac{a_{1}}{2}+\frac{a_{2}}{2}+\frac{i}{2}\right)} \\
& \times \frac{\Gamma\left(2 b_{1}+2 b_{1}^{\prime}-\frac{a_{1}}{2}-\frac{a_{2}}{2}+\frac{i}{2}+j+\frac{1}{2}\right) \Gamma\left(b_{1}+b_{1}^{\prime}+j+\frac{\left(-a_{1}+a_{2}+i+1\right)}{2}\right)}{\Gamma\left(2 b_{1}+2 b_{1}^{\prime}-\frac{a_{1}}{2}-\frac{a_{2}}{2}+\frac{i}{2}+j+\frac{1}{2}\right)} \\
& \times \frac{\Gamma\left(\frac{1}{2}-\frac{a_{1}}{2}+\frac{a_{2}}{2}-\frac{|i|}{2}\right) \Gamma\left(b_{1}+b_{1}^{\prime}-\frac{a_{1}}{2}-\frac{a_{2}}{2}+\frac{j}{2}+\frac{1}{2}-\frac{|i+j|}{2}\right)}{\Gamma\left(b_{1}+b_{1}^{\prime}-\frac{a_{1}}{2}+\frac{a_{2}}{2}+\frac{i}{2}+j+\frac{1}{2}\right)} \\
& \times\left\{A_{i j} \frac{\Gamma\left(\frac{a_{1}}{2}-\frac{j}{2}-1+\left[\frac{j+1}{2}\right]\right) \Gamma\left(\frac{a_{2}}{2}-\frac{i}{2}-\frac{j}{2}+\left[\frac{i+j+1}{2}\right]\right)}{\Gamma\left(b_{1}+b_{1}^{\prime}+\frac{j}{2}-\frac{a_{1}}{2}+\frac{1}{2}\right) \Gamma\left(b_{1}+b_{1}^{\prime}-\frac{a_{2}}{2}-\frac{i}{2}+\frac{j}{2}+\frac{1}{2}+\left[\frac{i}{2}\right]\right)}\right.
\end{aligned}
$$


572 Yong Sup Kim, Arjun K. Rathie, Chan Bong Park and Chang Hyun Lee

$$
\left.+B_{i j} \frac{\Gamma\left(\frac{a_{1}}{2}-\frac{j}{2}+\frac{1}{2}+\left[\frac{j}{2}\right]\right) \Gamma\left(\frac{a_{2}}{2}-\frac{i}{2}-\frac{j}{2}+\frac{1}{2}+\left[\frac{i+j}{2}\right]\right)}{\Gamma\left(b_{1}+b_{1}^{\prime}+\frac{j}{2}-\frac{a_{1}}{2}\right) \Gamma\left(b_{1}+b_{1}^{\prime}-\frac{a_{2}}{2}-\frac{i}{2}+\frac{j}{2}+\left[\frac{i+1}{2}\right]\right)}\right\}
$$

for $i=-3,-2,-1,0,1,2$ and $j=0,1,2,3$.

The coefficients $A_{i, j}$ and $B_{i, j}$ can be obtained from the tables of $A_{i j}$ and $B_{i j}$ given at the end of this paper by replacing $a$ by $2 b_{1}+2 b_{1}^{\prime}-a_{1}+j$, $b$ by $\frac{1}{2}-\frac{1}{2} a_{1}+\frac{1}{2} a_{2}+\frac{1}{2} i$ and $c$ by $b_{1}+b_{1}^{\prime}-\frac{1}{2} a_{1}-\frac{1}{2} a_{2}+\frac{1}{2} i+j+\frac{1}{2}$, respectively.

\section{Derivation}

First we shall prove that

$$
\begin{aligned}
& F_{2: 0 ; 0}^{2: 1 ; 1}\left[\begin{array}{l}
a_{1}, a_{2}: b_{1}, b_{1}^{\prime} \\
c_{1}, c_{2}:-,-
\end{array}\right] \\
& =\frac{\Gamma\left(c_{1}\right) \Gamma\left(c_{2}\right) \Gamma\left(c_{1}+c_{2}-a_{1}-a_{2}-b_{1}-b_{1}^{\prime}\right)}{\Gamma\left(a_{1}\right) \Gamma\left(c_{1}+c_{2}-a_{1}-a_{2}\right) \Gamma\left(c_{1}+c_{2}-a_{1}-b_{1}-b_{1}^{\prime}\right)} \\
& \times{ }_{3} F_{2}\left[\begin{array}{c}
c_{1}-a_{1}, c_{2}-a_{1}, c_{1}+c_{2}-a_{1}-a_{2}-b_{1}-b_{1}^{\prime} ; \\
c_{1}+c_{2}-a_{1}-a_{2}, c_{1}+c_{2}-a_{1}-b_{1}-b_{1}^{\prime} ;
\end{array}\right]
\end{aligned}
$$

Proof. By noting $(\alpha)_{m+n}=(\alpha+m)_{n}(\alpha)_{m}$ and starting with (1.1), the left hand side of (4.1) is

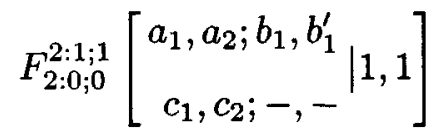

$$
\begin{aligned}
& =\sum_{m=0}^{\infty} \sum_{n=0}^{\infty} \frac{\left(a_{1}\right)_{m+n}\left(a_{2}\right)_{m+n}\left(b_{1}\right)_{m}\left(b_{1}^{\prime}\right)_{n}}{\left(c_{1}\right)_{m+n}\left(c_{2}\right)_{m+n} m ! n !} \\
& =\sum_{m=0}^{\infty} \sum_{n=0}^{\infty} \frac{\left(a_{1}+m\right)_{n}\left(a_{1}\right)_{m}\left(a_{2}+m\right)_{n}\left(a_{2}\right)_{m}\left(b_{1}\right)_{m}\left(b_{1}^{\prime}\right)_{n}}{\left(c_{1}+m\right)_{n}\left(c_{1}\right)_{m}\left(c_{2}+m\right)_{n}\left(c_{2}\right)_{m} m ! n !} \\
& =\sum_{m=0}^{\infty} \frac{\left(a_{1}\right)_{m}\left(a_{2}\right)_{m}\left(b_{1}\right)_{m}}{\left(c_{1}\right)_{m}\left(c_{2}\right)_{m} m !} \sum_{n=0}^{\infty} \frac{\left(a_{1}+m\right)_{n}\left(a_{2}+m\right)_{n}\left(b_{1}^{\prime}\right)_{n}}{\left(c_{1}+m\right)_{n}\left(c_{2}+m\right)_{n} n !} \\
& =\sum_{m=0}^{\infty} \frac{\left(a_{1}\right)_{m}\left(a_{2}\right)_{m}\left(b_{1}\right)_{m}}{\left(c_{1}\right)_{m}\left(c_{2}\right)_{m} m !} F_{2}\left[\begin{array}{c}
a_{1}+m, a_{2}+m, b_{1}^{\prime} \\
c_{1}+m, c_{2}+m
\end{array}\right]
\end{aligned}
$$


Now using the second fundamental transformation formula (2.1), we get

$$
\begin{aligned}
= & \sum_{m=0}^{\infty} \frac{\left(a_{1}\right)_{m}\left(a_{2}\right)_{m}\left(b_{1}\right)_{m}}{\left(c_{1}\right)_{m}\left(c_{2}\right)_{m} m !} \\
& \times \frac{\Gamma\left(c_{1}+m\right) \Gamma\left(c_{2}+m\right) \Gamma\left(c_{1}+c_{2}-a_{1}-a_{2}-b_{1}^{\prime}\right)}{\Gamma\left(a_{1}+m\right) \Gamma\left(c_{1}+c_{2}-a_{1}-b_{1}^{\prime}+m\right) \Gamma\left(c_{1}+c_{2}-a_{1}-a_{2}\right)} \\
& \times{ }_{3} F_{2}\left[\begin{array}{c}
\left.c_{1}-a_{1}, c_{2}-a_{1}, c_{1}+c_{2}-a_{1}-a_{2}-b_{1}^{\prime} \mid 1\right] \\
c_{1}+c_{2}-a_{1}-b_{1}^{\prime}+m, c_{1}+c_{2}-a_{1}-a_{2}
\end{array}\right] \\
= & \frac{\Gamma\left(c_{1}\right) \Gamma\left(c_{2}\right) \Gamma\left(c_{1}+c_{2}-a_{1}-a_{2}-b_{1}^{\prime}\right)}{\Gamma\left(a_{1}\right) \Gamma\left(c_{1}+c_{2}-a_{1}-b_{1}^{\prime}\right) \Gamma\left(c_{1}+c_{2}-a_{1}-a_{2}\right)} \\
& \times \sum_{m=0}^{\infty} \frac{\left(a_{2}\right)_{m}\left(b_{1}\right)_{m}}{m !\left(c_{1}+c_{2}-a_{1}-b_{1}^{\prime}\right)_{m}} \\
& \times{ }_{3} F_{2}\left[\begin{array}{c}
c_{1}-a_{1}, c_{2}-a_{1}, c_{1}+c_{2}-a_{1}-a_{2}-b_{1}^{\prime} \\
c_{1}+c_{2}-a_{1}-b_{1}^{\prime}+m, c_{1}+c_{2}-a_{1}-a_{2}
\end{array}\right] \\
= & \frac{\Gamma\left(c_{1}\right) \Gamma\left(c_{2}\right) \Gamma\left(c_{1}+c_{2}-a_{1}-a_{2}-b_{1}^{\prime}\right)}{\Gamma\left(a_{1}\right) \Gamma\left(c_{1}+c_{2}-a_{1}-b_{1}^{\prime}\right) \Gamma\left(c_{1}+c_{2}-a_{1}-a_{2}\right)} \\
& \times \sum_{m=0}^{\infty} \sum_{n=0}^{\infty} \frac{\left(a_{2}\right)_{m}\left(b_{1}\right)_{m}}{m !\left(c_{1}+c_{2}-a_{1}-b_{1}^{\prime}\right)_{m}} \\
& \times \frac{\left(c_{1}-a_{1}\right)_{n}\left(c_{2}-a_{1}\right)_{n}\left(c_{1}+c_{2}-a_{1}-a_{2}-b_{1}^{\prime}\right)_{n}}{\left(c_{1}+c_{2}-a_{1}-b_{1}^{\prime}+m\right)_{n}\left(c_{1}+c_{2}-a_{1}-a_{2}\right)_{n} n !} \\
& \left.\times{ }_{i}\right)
\end{aligned}
$$

But it is easy to see that

$$
\begin{aligned}
& \left(c_{1}+c_{2}-a_{1}-b_{1}^{\prime}+m\right)_{n}\left(c_{1}+c_{2}-a_{1}-b_{1}^{\prime}\right)_{m} \\
= & \left(c_{1}+c_{2}-a_{1}-b_{1}^{\prime}+n\right)_{m}\left(c_{1}+c_{2}-a_{1}-b_{1}^{\prime}\right)_{n} \\
& F_{2: 0 ; 0}^{2: 1 ; 1}\left[\begin{array}{l}
a_{1}, a_{2}: b_{1}, b_{1}^{\prime} \\
\left.c_{1}, c_{2}:-,-1,1\right]
\end{array}\right. \\
= & \frac{\Gamma\left(c_{1}\right) \Gamma\left(c_{2}\right) \Gamma\left(c_{1}+c_{2}-a_{1}-a_{2}-b_{1}^{\prime}\right)}{\Gamma\left(a_{1}\right) \Gamma\left(c_{1}+c_{2}-a_{1}-b_{1}^{\prime}\right) \Gamma\left(c_{1}+c_{2}-a_{1}-a_{2}\right)} \\
& \times \sum_{m=0}^{\infty} \sum_{n=0}^{\infty} \frac{\left(a_{2}\right)_{m}\left(b_{1}\right)_{m}}{m !\left(c_{1}+c_{2}-a_{1}-b_{1}^{\prime}+n\right)_{m}} \\
& \times \frac{\left(c_{1}-a_{1}\right)_{n}\left(c_{2}-a_{1}\right)_{n}\left(c_{1}+c_{2}-a_{1}-a_{2}-b_{1}^{\prime}\right)_{n}}{\left(c_{1}+c_{2}-a_{1}-b_{1}^{\prime}\right)_{n}\left(c_{1}+c_{2}-a_{1}-a_{2}\right)_{n} n !}
\end{aligned}
$$


574 Yong Sup Kim, Arjun K. Rathie, Chan Bong Park and Chang Hyun Lee

$$
\begin{aligned}
= & \frac{\Gamma\left(c_{1}\right) \Gamma\left(c_{2}\right) \Gamma\left(c_{1}+c_{2}-a_{1}-a_{2}-b_{1}^{\prime}\right)}{\Gamma\left(a_{1}\right) \Gamma\left(c_{1}+c_{2}-a_{1}-b_{1}^{\prime}\right) \Gamma\left(c_{1}+c_{2}-a_{1}-a_{2}\right)} \\
& \times \sum_{n=0}^{\infty} \frac{\left(c_{1}-a_{1}\right)_{n}\left(c_{2}-a_{1}\right)_{n}\left(c_{1}+c_{2}-a_{1}-a_{2}-b_{1}^{\prime}\right)_{n}}{\left(c_{1}+c_{2}-a_{1}-b_{1}^{\prime}\right)_{n}\left(c_{1}+c_{2}-a_{1}-a_{2}\right)_{n} n !} \\
& \times \sum_{m=0}^{\infty} \frac{\left(a_{2}\right)_{m}\left(b_{1}\right)_{m}}{\left(c_{1}+c_{2}-a_{1}-b_{1}^{\prime}+n\right)_{m} m !} \\
= & \frac{\Gamma\left(c_{1}\right) \Gamma\left(c_{2}\right) \Gamma\left(c_{1}+c_{2}-a_{1}-a_{2}-b_{1}^{\prime}\right)}{\Gamma\left(a_{1}\right) \Gamma\left(c_{1}+c_{2}-a_{1}-b_{1}^{\prime}\right) \Gamma\left(c_{1}+c_{2}-a_{1}-a_{2}\right)} \\
& \times \sum_{n=0}^{\infty} \frac{\left(c_{1}-a_{1}\right)_{n}\left(c_{2}-a_{1}\right)_{n}\left(c_{1}+c_{2}-a_{1}-a_{2}-b_{1}^{\prime}\right)_{n}}{\left(c_{1}+c_{2}-a_{1}-b_{1}^{\prime}\right)_{n}\left(c_{1}+c_{2}-a_{1}-a_{2}\right)_{n} n !} \\
& \times a_{2}, b_{1} F_{1}\left[\begin{array}{c}
\mid 1 \\
c_{1}+c_{2}-a_{1}-b_{1}^{\prime}+n
\end{array}\right] .
\end{aligned}
$$

Using the well-known Gauss's theorem [1, p. 2], we have

$$
\begin{aligned}
= & \frac{\Gamma\left(c_{1}\right) \Gamma\left(c_{2}\right) \Gamma\left(c_{1}+c_{2}-a_{1}-a_{2}-b_{1}^{\prime}\right)}{\Gamma\left(a_{1}\right) \Gamma\left(c_{1}+c_{2}-a_{1}-b_{1}^{\prime}\right) \Gamma\left(c_{1}+c_{2}-a_{1}-a_{2}\right)} \\
& \times \sum_{n=0}^{\infty} \frac{\left(c_{1}-a_{1}\right)_{n}\left(c_{2}-a_{1}\right)_{n}\left(c_{1}+c_{2}-a_{1}-a_{2}-b_{1}^{\prime}\right)_{n}}{\left(c_{1}+c_{2}-a_{1}-b_{1}^{\prime}\right)_{n}\left(c_{1}+c_{2}-a_{1}-a_{2}\right)_{n} n !} \\
& \times \frac{\Gamma\left(c_{1}+c_{2}-a_{1}-b_{1}^{\prime}+n\right) \Gamma\left(c_{1}+c_{2}-a_{1}-a_{2}-b_{1}-b_{1}^{\prime}+n\right)}{\Gamma\left(c_{1}+c_{2}-a_{1}-b_{1}-b_{1}^{\prime}+n\right) \Gamma\left(c_{1}+c_{2}-a_{1}-a_{2}-b_{1}^{\prime}+n\right)} \\
= & \frac{\Gamma\left(c_{1}\right) \Gamma\left(c_{2}\right) \Gamma\left(c_{1}+c_{2}-a_{1}-a_{2}-b_{1}^{\prime}\right)}{\Gamma\left(a_{1}\right) \Gamma\left(c_{1}+c_{2}-a_{1}-a_{2}\right) \Gamma\left(c_{1}+c_{2}-a_{1}-b_{1}-b_{1}^{\prime}\right)} \\
& \times \sum_{n=0}^{\infty} \frac{\left(c_{1}-a_{1}\right)_{n}\left(c_{2}-a_{1}\right)_{n}\left(c_{1}+c_{2}-a_{1}-a_{2}-b_{1}-b_{1}^{\prime}\right)_{n}}{\left(c_{1}+c_{2}-a_{1}-a_{2}\right)_{n}\left(c_{1}+c_{2}-a_{1}-b_{1}-b_{1}^{\prime}\right)_{n} n !} \\
= & \frac{\Gamma\left(c_{1}\right) \Gamma\left(c_{2}\right) \Gamma\left(c_{1}+c_{2}-a_{1}-a_{2}-b_{1}-b_{1}^{\prime}\right)}{\Gamma\left(a_{1}\right) \Gamma\left(c_{1}+c_{2}-a_{1}-a_{2}\right) \Gamma\left(c_{1}+c_{2}-a_{1}-b_{1}-b_{1}^{\prime}\right)} \\
& \times{ }_{3} F_{2}\left[\begin{array}{c}
c_{1}-a_{1}, c_{2}-a_{1}, c_{1}+c_{2}-a_{1}-a_{2}-b_{1}-b_{1}^{\prime} \\
c_{1}+c_{2}-a_{1}-a_{2}, c_{1}+c_{2}-a_{1}-b_{1}-b_{1}^{\prime}
\end{array}\right] .
\end{aligned}
$$

So we arrive at the right hand side of (4.1). This completes the proof of (4.1).

Now, in (4.1), if we take $c_{1}=2 b_{1}+2 b_{1}^{\prime}+j$ and $c_{2}=\frac{1}{2}\left(1+a_{1}+a_{2}+i\right)$, 
then for $i=0, \pm 1, \pm 2, \pm 3$ and $j=1,2,3$ we have

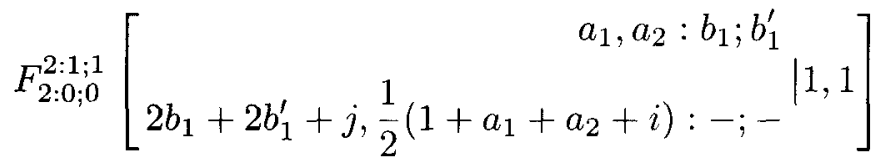

$$
\begin{aligned}
& =\frac{\Gamma\left(2 b_{1}+2 b_{1}^{\prime}+j\right) \Gamma\left(\frac{1}{2}+\frac{a_{1}}{2}+\frac{a_{2}}{2}+\frac{i}{2}\right)}{\Gamma\left(a_{1}\right) \Gamma\left(2 b_{1}+2 b_{1}^{\prime}-\frac{a_{1}}{2}-\frac{a_{2}}{2}+\frac{i}{2}+j+\frac{1}{2}\right)} \\
& \times \frac{\Gamma\left(b_{1}+b_{1}^{\prime}-\frac{a_{1}}{2}-\frac{a_{2}}{2}+\frac{i}{2}+j+\frac{1}{2}\right)}{\Gamma\left(b_{1}+b_{1}^{\prime}-\frac{a_{1}}{2}+\frac{a_{2}}{2}+\frac{i}{2}+j+\frac{1}{2}\right)} \\
& \times_{3} F_{2}\left[\begin{array}{r}
2 b_{1}+2 b_{1}^{\prime}+j-a_{1}, A, b_{1}+b_{1}^{\prime}+j+B \\
2 b_{1}+2 b_{1}^{\prime}+j+B, b_{1}+b_{1}^{\prime}+A
\end{array}\right],
\end{aligned}
$$

where $A=\frac{\left(-a_{1}+a_{2}+i+1\right)}{2}$ and $B=\frac{\left(-a_{1}-a_{2}+i+1\right)}{2}$.

Now the ${ }_{3} F_{2}$ on the right-hand side can be evaluated with the help of generalized Dixon's theorem (2.2) and after some simplification, we arrive at the desired result (3.1).

For $i=j=0$, we get a known result due to Bhatt and Pandey's formula (1.2).

\section{References}

[1] W. N. Bailey, Gerneralized Hypergeometric series, Cambridge Tracts in Math., 1935 Reprinted by Stechert-Hafner, New York, 1964.

[2] R. C. Bhatt and R. C. Pandey, On certain sums and transformations of hypergeometric functions of two variables of superior order, Ganita 16 (1965), 89-98.

[3] J. Kampé de Fériet, Les Fonctions hypergéométriques d'ordre supérieur à deux variables, C. R. Acad. Sci. Paris 173 (1921), 401-404.

[4] J. L. Lavoie, F. Grondin, A. K. Rathie and K. Arora, Generalizations of Dixon's theorem on the sum of $a_{3} F_{2}$, Math. Comp. 62 (1994), 267-276.

[5] H. M. Srivastava and R. Panda, An integral representation for the product of two Jacobi polynomials, J. London Math. Soc. (2) 12 (1976), 419-425.

Yong Sup Kim

Department of Mathematics

Wonkwang University

Iksan 570-749, Korea

E-mail: yspkim@wonkwang.ac.kr 
Arjun K. Rathie

Department of Mathematics

Dungar College (Bikaner University)

Bikaner-334001, Rajasthan State, India

E-mail: akrathie@rediffmail.com

Chan Bong Park

Department of Mathematics

Wonkwang University

Iksan 570-749, Korea

E-mail: cbpark@wonkwang.ac.kr

Chang Hyun Lee

Department of Mathematics

Seonam University

Namwon 590-711, Korea

E-mail: chlee@seonam.ac.kr 